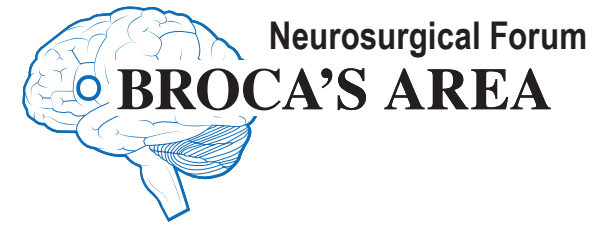

\section{Developmental stages in the career of an academic neurosurgeon}

\author{
Ralph G. Dacey Jr., MD \\ Department of Neurological Surgery, Washington University School of \\ Medicine, St. Louis, Missouri
}

$\mathrm{T}$ There is value in examining and describing the developmental stages in the career of an academic neurosurgeon.

This developmental evolution can be considered across three domains: clinical neurosurgery, research, and leadership. Academic neurosurgeons must educate successive generations, and I will discuss the importance of teaching for each domain. Personal and family development and concerns are probably the most important for all of us as we progress through our lives, but these are so individual that I will not consider them as a separate domain. This is not a discussion of retirement or end-of-career issues because that is a separate topic in and of itself.

We can consider the developmental progress that occurs as the neurosurgeon evolves from resident to assistant professor to associate professor to full professor. The matrix of developmental stages in the clinical, research, and leadership domains is shown in Fig. 1, and the stages are described in further detail below. The relative importance of the various domains changes over the stages of an academic neurosurgeon's career (Fig. 2). For example, when a neurosurgeon is an assistant professor, research may take up more of his or her time and interest, but with the transition to the associate professor and professor levels, clinical practice may become much more prominent for some individuals. For others, clinical practice is the dominant aspect of their neurosurgical career from the outset, but a part of their career that itself undergoes a natural evolution over time-from establishing local technical expertise to advancing the field through surgical innovation to attaining a regional/national reputation as a master technical surgeon. For many neurosurgeons, a role as local, regional, and/or national leader also evolves as their career progresses (Fig. 3). Between the developmental stages there are predictable times of turbulence and challenge that could be described as developmental countercurrents. The degree to which individual neurosurgeons focus their efforts on clinical neurosurgery or on research varies significantly, and many academic neurosurgeons conduct successful careers concentrating on one of these two domains.

In this paper, I will try to describe the common distinctive characteristics of the stages in development. I will discuss conflicts and tensions that often come up during transitions between developmental stages.

\section{Development in the Clinical Domain}

The most important aspect of career development for an academic neurosurgeon usually occurs in the clinical domain. Initially-usually in PGY 1 through PGY 4-the neurosurgery resident is in a phase of adaptive enculturation. During this phase, the resident watches how neurosurgical care is delivered and procedures are conducted and imitates the activities of his/her mentors and teachers. $\mathrm{He}$ or she learns the rules and norms of our specialty and begins the assimilation of basic knowledge about anatomy, surgical care, neurology, and the fundamental characteristics of clinical neurosurgical disease. The resident must adapt personally to the culture of neurosurgery, and sometimes this is difficult and challenging. During this phase of adaptive enculturation, the neurosurgical resident, although becoming progressively more autonomous, is not independent for most important clinical decision-making.

During the next phase of clinical career development, usually occurring from PGY 5 through PGY 7, the resident enters a phase of autonomous integration into our specialty. The resident at this stage begins to "own" the patient and enjoys the successes of satisfactory treatment and feels the failure of complications when they occur. During this stage, the resident becomes broadly competent and relatively autonomous. He or she experiences the value of the cross-pollination of various neurosurgical subspecialties (vascular, tumor, spine, etc.) and a rapid acceleration of clinical expertise. Residents must also progressively 
Clinical

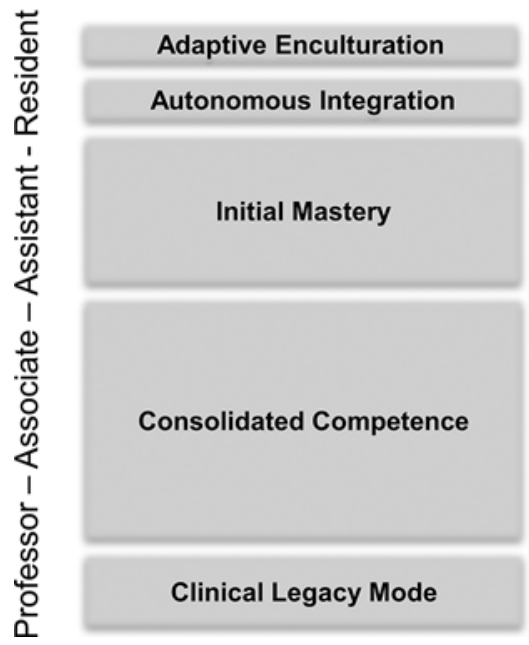

Research

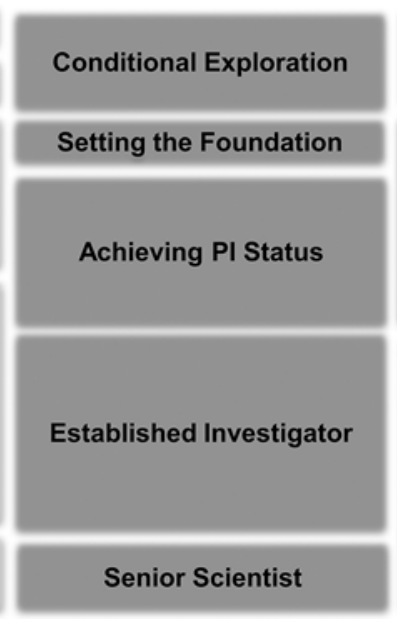

Leadership

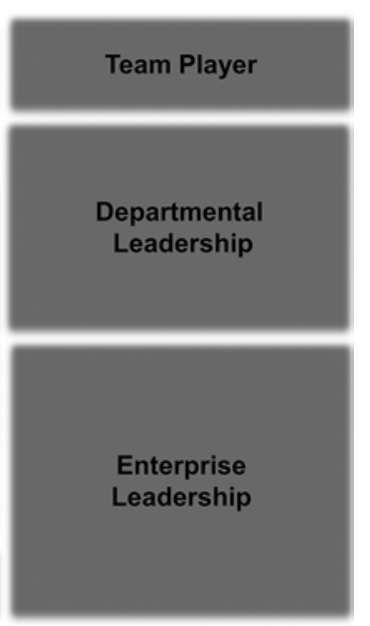

FIG. 1. Stages of career development for academic neurosurgeons within the clinical, research, and leadership domains as they progress through their careers from resident to professor. The amount of effort in the various domains may change at various stages of development.

develop physical stamina to perform long operative procedures and emotional resilience to deal with very sick patients under pressure. Almost all residents develop a great sense of pride, confidence, and accomplishment during this stage of autonomous integration.

As the neurosurgical resident goes through this progression from enculturation to autonomy, it can be difficult to simultaneously maintain adherence to our specialty's strict expectations and norms in terms of quality and independence. Some residents are slow to become autonomous, and their autonomous performance of neurosurgical duties (especially in the operating room) needs to be tempered by judgment and social proprioception. As a result of the expectations of patients, payers, and hospital administrators, our training paradigms in the United States do not always foster independence. However, the development of autonomy is fundamental to achieving that level of confidence that we all recognize as essential for a successful transition from senior resident to independent neurosurgical practitioner. It is a difficult and challenging progression, and it is often associated with significant pressure on the individual and tension in his or her interactions with coworkers and supervisors.

At the next stage within the clinical domain, the neurosurgeon becomes an assistant professor. They begin to explore their own limits and define a clinical interest. This is the phase of initial mastery. They accept challenges, they develop tolerance for risk, and they define their own clinical style. Usually, this phase is characterized by progressively increasing surgical volume, which in turn generates more volume and practice opportunities. Also at this time, it is difficult to establish work/life balance, as personal and family issues often peak as the neurosurgeon enters his or her late 30s. The importance of self-discovery as a surgeon is characterized by distinguishing ourselves from those around us and determining what is important to us. The neurosurgeon must experiment and learn by trial and error. Some of the things that we try do not go well, and we learn our limitations. If a neurosurgeon becomes "stuck" at this stage and cannot discover himself or herself as a surgeon, he or she generally cannot go on to the next stage. This phenomenon is, however, unusual.

The developmental countercurrent that occurs around this stage relates to the tension between patient safety and caution versus the need to innovate and challenge surgical boundaries. Establishing the right balance between caution and prudence and developing stretch goals for surgical growth define this tension. It is important that the culture of the institution, group, or department be tuned to promote the proper balance. During the period of initial mastery, the more junior surgeon must be actively supported in practice and skill development by his/her more senior surgical colleagues. As an example, I recently treated a patient who presented with acromegaly. As we were considering an endonasal endoscopic transsphenoidal approach for surgical treatment of the offending adenoma, it became clear that we could not properly manipulate the endoscope due to the patient's profound cervical kyphosis (chin-on-chest deformity). I therefore asked one of my colleagues to perform a deformity correction procedure that would restore a relatively normal cervical alignment for the patient, allowing her to undergo endonasal endoscopic transsphenoidal resection of the pituitary adenoma causing her acromegaly. This was a complicated set of procedures for this patient, but ultimately it was very successful. As my younger colleague addressed the cervical deformity, it was clear that the procedure would be complex and fraught with significant risk. My colleague accepted this, dealt with it, and successfully brought the patient through a complex procedure. This and other cases like it have led to his developing broad competence in managing complex spinal deformity patients and gaining great confidence in choosing the indications and best course for his patients.

Also at this time the neurosurgeon must develop the proper balance between grit as described by Angela Duckworth $^{2}$ and flow as described by Mihaly Csikszentmihalyi. ${ }^{1}$ Grit is manifested by persistence and resilience and has to be part of every neurosurgeon's makeup. Grit is needed to 


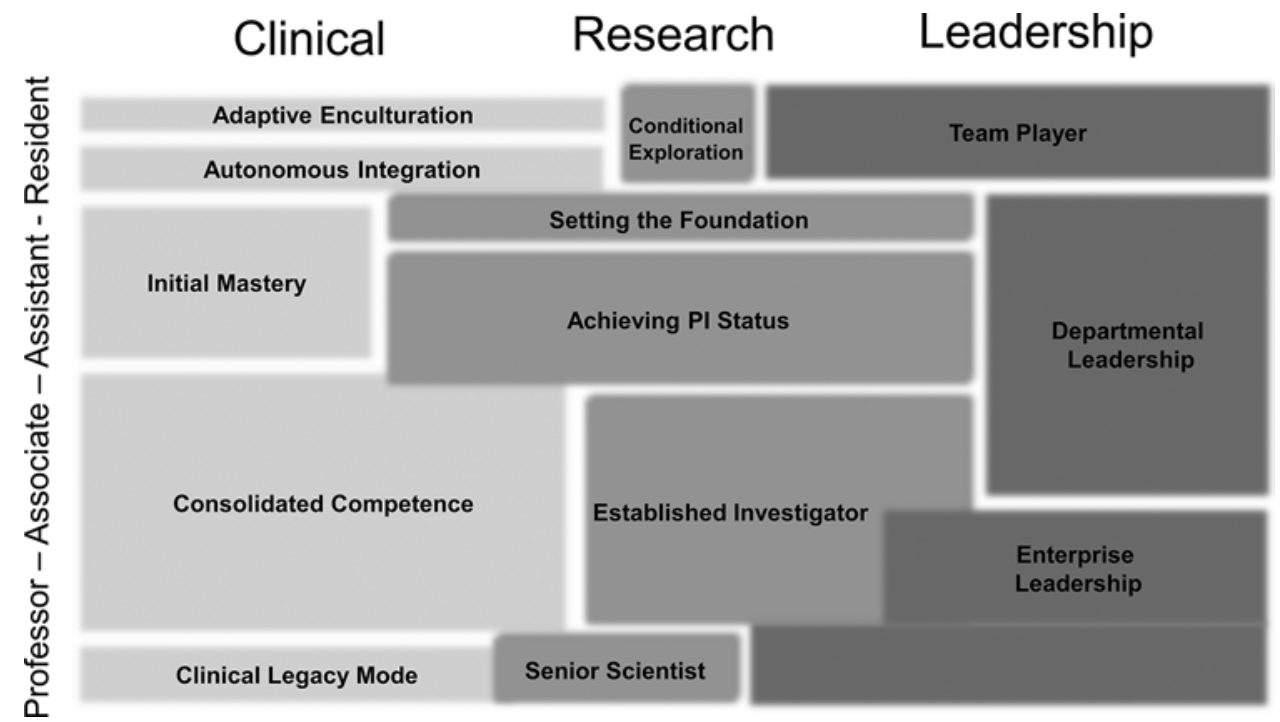

FIG. 2. Stages of career development model adjusted to illustrate the change in the relative importance of the domains as the neurosurgeon becomes an assistant professor and spends more time in research.

spend hours performing tedious micro-dissection to safely remove a large foramen magnum meningioma. Flow is the result of practice and subconscious skill development, which are also prominent features in the characteristics of a successful neurosurgeon at this stage. Flow occurs when an experienced neurosurgeon intuitively knows exactly which clip will completely occlude a large aneurysm. A powerful combination of grit and flow is present in most neurosurgeons.

The next stage in career development could be called consolidated competence. During this stage, the associate and full professor progressively develops expertise in managing increasingly complex clinical challenges. The neurosurgeon is adept at identifying and avoiding "failure modes." For example, in managing a parietal parasagittal meningioma, he or she has become an expert in avoiding the complication of damage to the superior sagittal sinus, which could be associated with a fatal outcome or severe neurological disability. The clinical focus of the neurosurgeon often narrows while overall surgical volume increases, which often leads to some pruning of his or her clinical interests and scope of practice. Referral patterns also tend to concentrate surgical volume as the neurosurgeon becomes more prominent. In some areas, there may be an increase in research activity especially as it complements a growing clinical practice. The neurosurgeon at this stage is frequently "paying it forward" in terms of supporting the practice development of his or her more junior colleagues-an important cultural aspect of the group or department in many cases. As the neurosurgeon in this stage becomes more prominent in the community, there is recognition of his or her preeminence, and the neurosurgeon is often asked to lead more in the hospital, the medical school, and the community.

The last stage of clinical development is the clinical legacy mode. A number of prominent neurosurgeons over the years (e.g., Thor Sundt, John Jane, Robert Spetzler) have had huge international legacies as a result of their academic and educational contributions. But even for most neurosurgeons at this stage of their career, a large group of grateful patients, families, and colleagues express appreciation, which leads to progressively increasing satisfaction with the role of being an academic neurosurgeon. This satisfaction also is associated with a recognition by most neurosurgeons of the enormous gifts that we can give to others, especially our patients, our trainees, our friends, and our colleagues. To be a neurosurgeon at this stage is to serve others, and despite the demands that this places on the personal life of a neurosurgeon, it is extremely rewarding. Senior neurosurgeons spend a lot of time supporting and advising their associates. They learn to enjoy the accomplishments of their colleagues, and patient care actually becomes even more satisfying and important to them. Figure 4 is a plot of the increasing effectiveness of practice versus a neurosurgeon's leadership skills. A person with low capabilities in both of these axes is relatively useless, and a person with an inefficient or ineffective practice who has good leadership skills is also not optimal. What we all want to evolve to is that person who has great leadership skills and is a very effective practitioner who becomes a servant neurosurgical leader (see below).

The teaching component of the clinical domain is of great importance. Most academic neurosurgeons treasure the academic environment, where great value is placed on imparting knowledge to residents and conducting scholarly collaboration with fellow faculty members. Education and scholarship always strengthen clinical programs. As academic neurosurgeons progress in their clinical development, their pedagogic skills grow. They are most successful when they tell residents "what they are thinking" as they are delivering clinical care in the operating room or outpatient setting. Technical nuances and judgment related to surgical indications are the most important content they can deliver.

\section{Development in the Research Domain}

For most neurosurgeons, the research part of their career development begins in residency. From PGY 1 


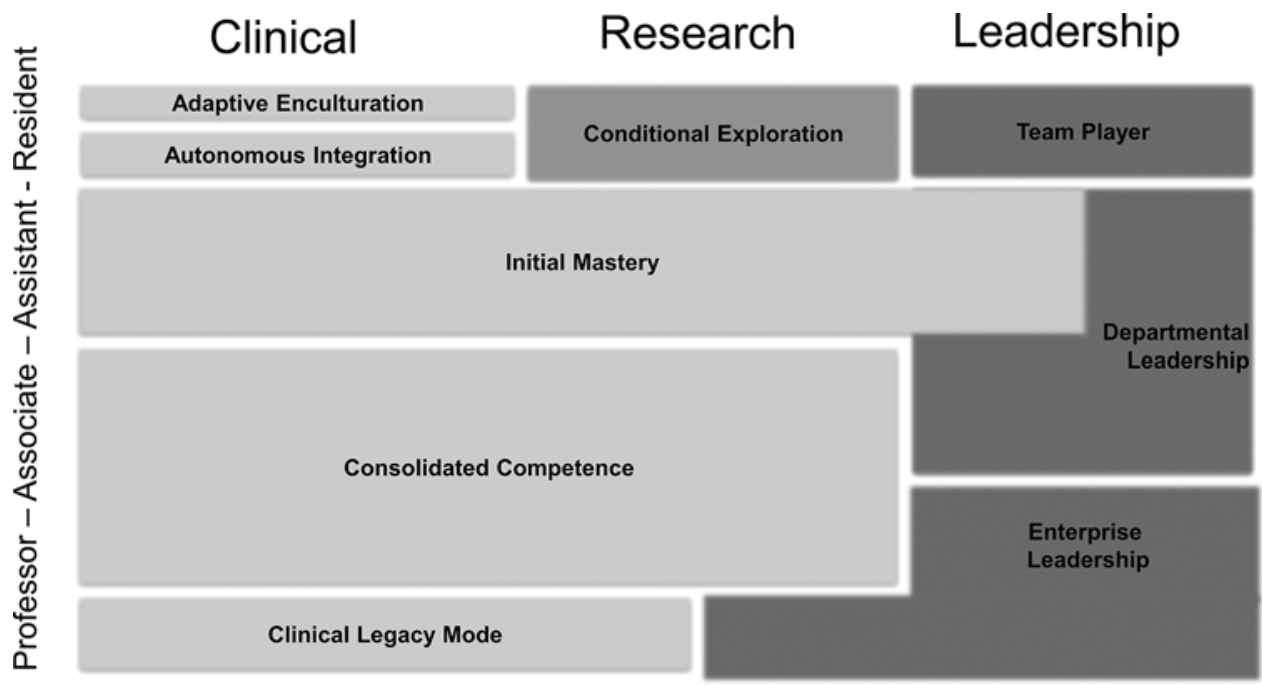

FIG. 3. Stages of career development model adjusted to illustrate evolving leadership. Some academic neurosurgeons concentrate on clinical practice, others primarily conduct research—both kinds of careers can lead to great contributions.

through PGY 4, their involvement with research consists of conditional exploration. They know that they will become neurosurgeons, but they are not sure whether they want to also become scientists/investigators. They must determine whether this would complement or conflict with their clinical interests and long-term career goals. The modes of research that they may become involved in could be basic, translational, or clinical or might involve device development and engineering or innovation.

Very early on in this stage, residents often note a countercurrent as the clash between the culture in the laboratory or research environment and the culture on the wards or in the operating room. The clinical culture is characterized by a commitment to patients' families and colleagues in the context of dealing with urgent or emergent clinical needs and everything that relates to that, including the likelihood of frequent and unpredictable interruptions in work and schedules. This contrasts with the laboratory culture, where sustained periods of focused concentration on scientific problems and experiments appear to be in conflict with the interruptions and unpredictability of the clinical schedule. Scientists and clinicians on either side of this divide may not have as much understanding or appreciation for the other culture.

The next stage in the research domain is setting the foundation. By this point, the neurosurgical resident has decided that he or she wishes to become a scientist in addition to being a neurosurgeon. They will often be participating in a postdoctoral fellowship. Their tasks here are to learn the scientific method from their mentors. They acquire a methodological set of tools (an understanding of molecular biology, facility with use of statistics to analyze data, etc.). They begin to network with other trainees and scientists at scientific meetings and seminars, and eventually they make some initial forays into independence as investigators.

When the neurosurgeon becomes an assistant professor, he/she is striving to achieve PI status. Many neurosurgeons start with a K-level career development award from the National Institutes of Health (NIH) to get started in their research careers as faculty members. The next several years are spent developing a coherent and forwardthinking research program that will ultimately translate to funding as a principal investigator (PI) on an R01 NIH research award. To do this, they must establish their independence as investigators, including balancing the need for continued mentorship while also separating themselves enough from their research mentors to successfully compete for an R-level NIH grant. Development of extensive collaborations across multiple research domains generally must also continue. Sometimes, this type of evolution can lead to negative reactions from former mentors, but it is an essential part of the development of the neurosurgeon.

The developmental countercurrent that occurs at this career stage is the potential conflict of the academic neurosurgeon's identity as a surgeon versus as a scientist. Scientists tend to be critical and skeptical and are rigorous about their own time management. They are searching for basic mechanisms, formulating hypotheses, and examining issues that have general scientific significance. Clinical surgeons, on the other hand, must be relatively non-judgmental, optimistic, and generous with giving time to patients and families. They usually take a practical approach to clinical problems and are comfortable working with relatively insufficient information. The contrast between these two identities can set up conflicts for neurosurgeons at this critical stage of their research careers. As they cycle back and forth between being in "scientific mode" versus "clinical mode," they can be challenged by colleagues on either side of the divide-by concern that they are not making fast enough progress in their scientific career and by the very significant clinical demands that neurosurgery places on its practitioners. It is important that the role of surgeon-scientist be appreciated and promoted within the academic department. In Fig. 5, surgical skill is plotted against scientific accomplishments. If someone has relatively poor surgical skills, but is scientifically accomplished, he or she is sometimes pejoratively referred to as a "rat doctor." Even in academic roles, many neurosurgeons find that they spend the overwhelming majority of their 


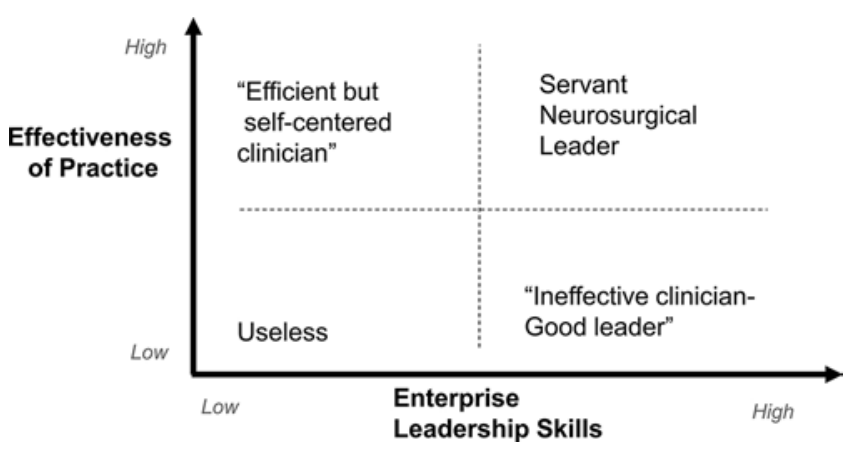

FIG. 4. Plot of effectiveness of practice versus skills demonstrated as an enterprise leader. We should all aspire to function in the quadrant of the servant neurosurgical leader.

time doing clinical work. Although this is for many people a satisfactory outcome, other neurosurgeons strive to become academic surgical leaders with both great scientific accomplishments and surgical skill.

A second countercurrent that can develop at this point is that it is sometimes difficult for neurosurgeons to translate their investigative work into meaningful research programs that truly impact the lives of patients. The hypercompetitive NIH culture can drive the scientist to constantly move into new areas of technical experimentation, but at the same time, the more that scientist learns about the system on which they are working, the narrower their perspective may become. The surgeon-scientist needs to determine at what point to continue to drive a relatively limited and focused concentration on the area in which he or she has become expert versus developing and consolidating a body of knowledge that is sustained by experiments that have a broad technological foundation and potential clinical application.

When neurosurgeons become associate or full professors, they enter into the developmental stage of an established investigator. Not all neurosurgeons want or are able to achieve this, but those that do attain this professional stage usually begin to be recognized locally and nationally for this achievement and often enter a research phase in which significant basic discoveries are made or preclinical findings begin to be translated into the clinic. This is also a stage when more junior colleagues seek mentorship and advice with establishment of their own investigative career-a request that is flattering but also anxiety-provoking, given the importance and responsibility associated with serving as an effective research mentor.

The senior surgeon-scientist is challenged to develop and maintain a research program characterized by a "grand theory" or the definition of a "coherent body of knowledge." The pressure to establish and continue a research program that strikes a balance between the need to discover fundamental mechanisms and/or translate fundamental findings to the clinic while remaining clinically relevant is the major challenge at this point. Another significant issue relates to managing the desire for adequate compensation as a senior neurosurgeon with more clinical activity versus continuing to spend a substantial amount of time in the lab with the associated impact on his or her compensation due to the "NIH pay scale."

The educational component of the research domain is

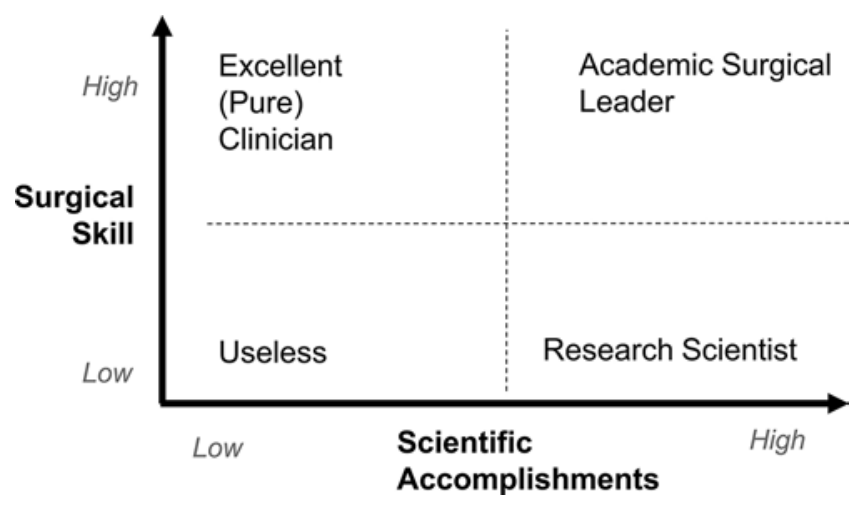

FIG. 5. Plot of surgical skill versus scientific accomplishments. Major contributions can be made in the quadrants of the excellent clinician and of the academic surgical leader.

a natural offshoot of the process of conducting research. As scientific hypotheses are being formulated and experiments are being designed, there is abundant opportunity to teach residents, graduate students, and postdoctoral fellows. The opportunity to continue to learn from investigators who are experienced and successful with regard to asking the right scientific questions is at a premium for students at all levels.

\section{Development in the Leadership Domain}

The third domain in which academic neurosurgeons evolve over the course of their professional life cycle is in their frequent desire to demonstrate and develop their capacity to lead. Leadership in this context is defined as "creating conditions in a group whereby the outcome of the team's activities is optimized."

Initially, successful residents are identified as team players as they become immersed in the neurosurgery culture. Their initial demonstrations of leadership behavior are "department-centric." They demonstrate loyalty, integrity, honesty, and reliability, and the development of these traits endears them to the faculty and their colleagues. They begin to become better leaders as they develop. It is important during this period that they demonstrate the ability to maintain composure under stress and equanimity in their dealings with other physicians and nurses. They also need to show that they can achieve good results working as part of a team of other residents. They represent the specialty and the neurosurgery department throughout the medical center, and this gives them additional opportunities to express leadership. One special condition that affects neurosurgeons because of the intensity of the work that they do is the need to maintain resilience while at the same time not letting their ability to be compassionate suffer from fatigue. Outbursts of anger or demeaning behavior may occur as a result of these pressures, but it is increasingly clear that this type of behavior has no place in the healthcare environment. As neurosurgeon-leaders develop, they need to be aware of the countercurrent related to the conflict between their identities as "lone surgical healers" versus "servant leaders." The neurosurgical culture often depends on residents and attending surgeons functioning as independent autonomous actors who "own" their results and are completely accountable. Neurosurgeons 


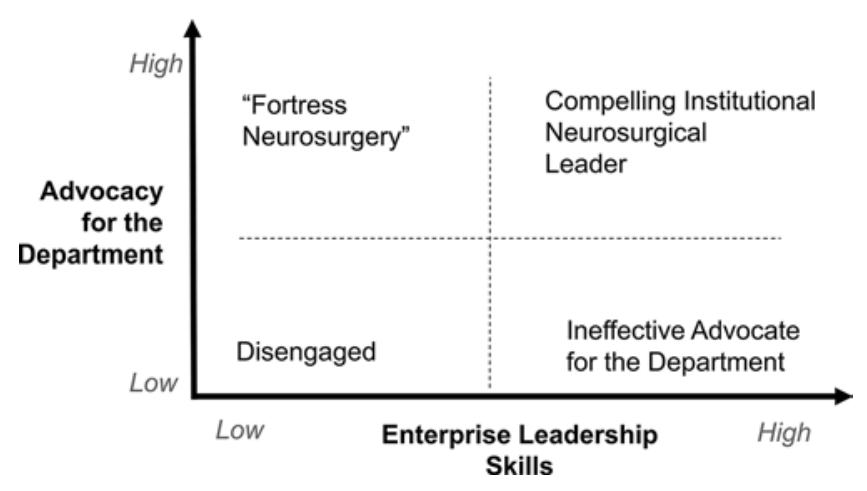

FIG. 6. Plot of advocacy for the department versus skills as an enterprise leader. Senior neurosurgical leaders should aspire to function in the quadrant of high advocacy for their department and great enterprise leadership skills.

tend to have less respect for others who do not have the same clinical values and degree of commitment to patient care in demanding and technically sophisticated contexts. Neurosurgeons know that they must strenuously advocate for their patients and their neurosurgical specialty to get the resources they need. However, if we want to become servant leaders within the broader institution, we must be careful not to let our particular culture poison the interactions that we have with other members of the medical school or hospital community.

The second stage in leadership development for most neurosurgeons is that they evolve from being team players as residents to departmental leaders as junior faculty members. They define an area of responsibility with regard to their colleagues in the department. They demonstrate that they can set and live up to high standards and they can balance other domains of development within their own career. They become firm and effective advocates for their own division, discipline, or department, and they set a clear vision for themselves in terms of their clinical administrative and research leadership. Leadership in this phase often involves the development of superior clinical programs, which may initially depend on the acquisition of sophisticated and expensive devices, such as the intraoperative MRI, the Gamma Knife, various types of surgical robots, and expensive operating microscopes. The extent to which a neurosurgical leader can facilitate the development of clinical programs determines, at this intermediate stage, the value that departmental colleagues place on the leadership skills of the individual.

The last stage of development for most neurosurgeons is that they evolve to become enterprise leaders. They may serve as division heads, department chairs or vice chairs, or program directors for the residency training program. In these roles, they must advocate for the broader enterprise of the medical school and hospital. They establish a balance between their acquisitiveness for their own interest and generosity for other programs and departments. The "package" that initially defines a leader's worth upon recruitment later becomes meaningless. The leaders' legacy status within the institution becomes the "package." Power becomes influence. Institutional leadership very often naturally extends into national leadership of professional and scientific organizations.

The countercurrent at this point is the fact that neurosurgical leaders need to continue to vigorously advocate for their own departments at the same time as they are becoming more prominent in the broader institution. It is necessary for the leader in this situation to oscillate between maintaining credibility as an institutional citizen versus having the appropriate degree of concern and attention to issues related to his or her program or department (Fig. 6). If advocacy for the department is not balanced by broader enterprise leadership skills, the neurosurgical leader is practicing "fortress neurosurgery" (John Jane, personal communication, 1983). When enterprise leadership skills match the skills that the neurosurgeon has for advocacy for his or her program, he or she becomes a compelling institutional neurosurgical leader with high credibility not only among fellow neurosurgeons, but also more widely in the institution. This is the endpoint that we should all strive to achieve.

Teaching others to lead is mostly done by example. Equanimity under stress is a quality that all trainees and young faculty recognize and try to emulate. It is important that young leaders learn to shift over time from advocating for their own interests to becoming effective in promoting the careers of their trainees and colleagues.

\section{Summary}

There are recognizable stages in the development of the career of an academic neurosurgeon. In their clinical, research, and leadership work, neurosurgeons progress sequentially toward more autonomy, better skills, and greater contributions. The transitions between the stages are often difficult; however, the gifts that we can give to others as neurosurgeons make our careers among the most rewarding that a human can undertake.

\section{Acknowledgments}

I am grateful to Ian Dorward, Gavin Dunn, Eric Leuthardt, Albert Kim, David Limbrick, Wilson Ray, Gregory Zipfel, and Jeanette Schillizzi for their advice and help in preparing the manuscript.

\section{References}

1. Csikszentmihalyi M: Flow: The Psychology of Optimal Experience. New York: Harper \& Row, 1990

2. Duckworth A: Grit: The Power of Passion and Perseverance. New York: Simon \& Schuster, 2016

\section{Disclosures}

The author reports no conflict of interest.

\section{Supplemental Information}

\section{Previous Presentations}

Part of this material was presented orally at the meeting of the Society of Neurological Surgeons, April 2017, Houston, Texas.

\section{Correspondence}

Ralph G. Dacey Jr.: daceyr@wustl.edu. 